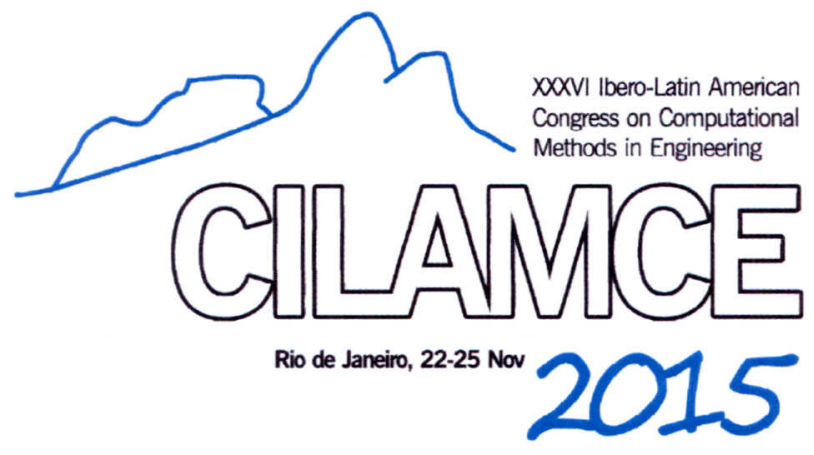

\title{
ANÁLISE DA ESTABILIDADE DE MACIÇOS SUBMARINOS SUBMETIDOS AO CARREGAMENTO CÍCLICO DA ONDA: UMA ABORDAGEM ANALÍTICO-NUMÉRICA
}

\author{
Deborah Marcant Silva Madalozzo \\ Samir Maghous \\ deborahmadalozzo@bol.com.br \\ samir.maghous@ufrgs.br
}

Universidade Federal do Rio grande do Sul

Av. Osvaldo Aranha, 99 - 3 andar, CEP 90035-190, Porto Alegre, RS, Brasil

Abstract. $O$ efeito das ondas de água sobre um leito submerso pode ser descrito pela propagação de uma onda de pressão ao longo de sua superfície. A teoria da análise limite permite formular condições suficientes e necessárias para a estabilidade do leito marinho em diferentes declividades. Em maciços constituídos por solos finos a análise da estabilidade é efetuada em condições não drenadas e utiliza-se o critério de Tresca para obtenção da capacidade de resistência da estrutura. Em leitos granulares, as tensões cisalhantes cíclicas podem causar uma densificação da estrutura do material, aumentando a pressão do fluido intersticial e diminuindo as tensões efetivas do material poroso. A capacidade de resistência é obtida através do critério de Mohr-Coulomb no contexto da validade do conceito das tensões efetivas de Terzaghi. O gradiente de poro-pressão atua como uma força volumétrica sobre o esqueleto e caracteriza o modo de carregamento principal da estrutura. No presente trabalho será utilizado um modelo simplificado que consiste em desacoplar o cálculo da poro-pressão e das deformações do esqueleto, a fim de considerar os comportamentos reversivel e irreversivel da estrutura porosa.

Keywords: Análise de estabilidade, Forças de percolação, Poroelasticidade, Poro-pressão, Ondas. 


\section{INTRODUÇÃO}

Devido ao desenvolvimento das estruturas marinhas, um crescente interesse sobre a resposta do leito do mar submetido ao carregamento da onda tem sido observado. Para águas rasas ou ondas fortes, a pressão da onda atuante sobre o leito do mar pode induzir deslocamentos significativos na massa de solo. Esse fenômeno pode causar o rompimento de dutos e deve ser levado em conta na análise de estabilidade de plataformas offshore, sendo grande importância para a engenharia costeira.

De forma geral, investigações sobre a resposta do leito do mar submetido ao carregamento da onda podem ser classificadas de acordo com o tipo de material que constitui o leito: um solo argiloso fino ou um solo granular. Diferenças essenciais aparecem na modelagem da resistência convencionalmente adotada para essas duas classes diferentes de material (Dormieux et al., 1993). Em solos granulares em especial, a pressão exercida pela onda sobre a face superior do maciço submarino é equilibrada por campos cíclicos de tensões e de pressão intersticial. Em razão da contractância das areias, ou seja, a tendência a se densificar quando submetidas a um estado de tensões desviadoras cíclicas, tal carregamento provoca em geral a acumulação de sobrepressões intersticiais no maciço. Nesse caso, o regime hidráulico é marcado por um fluxo de água intersticial nas zonas drenadas e, em uma análise de estabilidade, são as forças exercidas por esses fluxos sobre o esqueleto que caracterizam os esforços. Esse modo de carregamento do maciço, que leva em conta o caráter bifásico do solo, é distinto do considerado em solos coesivos, no qual a análise é realizada em tensões totais.

Para análise da estabilidade, a teoria da Analise Limite (Salençon 1983, 1990, 2013) pode fornecer condições suficientes e necessárias da estabilidade do leito marinho, as quais podem ser usadas para uma grande classe de carregamentos de onda e correspondentes distribuições de pressão. Em maciços constituídos por solos finos a capacidade resistente do material é geralmente modelada utilizando-se o critério de Tresca no contexto da análise não drenada (Dormieux, 1988; Dormieux, 1989; Dormieux \& Coussy, 1991; Dormieux, 1992). Por outro lado, em maciços constituídos por material granular, a capacidade resistente do material é geralmente modelada empregando-se o critério de Mohr-Coulomb, calculado sobre o tensor de tensões efetivas (Dormieux, 1989; Dormieux et al., 1993; De Buhan et al., 1999). Ainda no contexto da validade do conceito das tensões efetivas e utilizando a abordagem da Análise Limite, recentemente Saada et al (2012) apresentou um estudo da estabilidade de declives rochosos saturados de água.

O objetivo deste trabalho é investigar a estabilidade de maciços submarinos plano e levemente inclinado submetidos ao carregamento da onda no âmbito da teoria da Análise Limite, baseada em condições de deformações planas. Serão considerados maciços constituídos por material coesivo e granular e a pressão da onda sobre a superfície do maciço será modelada utilizando-se a teoria linearizada de Stokes. Para o cálculo da estabilidade em tensões efetivas será utilizada uma simplificação que consiste em desacoplar o cálculo da pressão intersticial, a fim de considerar separadamente os comportamentos reversível e irreversível da estrutura porosa.

\section{CARREGAMENTO DA ONDA DO MAR}

O carregamento da onda sobre o fundo do mar é assumido plano e sua forma é caracterizada por uma função $\mathrm{p}\left(\mathrm{x}_{1}, \mathrm{t}\right)$ e sua amplitude por um escalar positivo $\mathrm{p}_{0}$. Para a onda 
linear de Stokes, que assume fluxo irrotacional de fluido não viscoso e incompressível sobre leito horizontal e impermeável, a amplitude $\mathrm{p}_{0}$ é relacionada à profundidade da água $\mathrm{d}$ e às características geométricas da onda, isto é, o comprimento da onda L e à altura da onda $\mathrm{H}$ (a Fig. 1 apresentará estes parâmetros):

$$
p\left(x_{1}, t\right)=p_{0} \operatorname{sen}\left(k x_{1}-\omega t\right), \quad p_{0}=\frac{\gamma_{w} H}{2 \cosh (k d)} .
$$

onde $k=2 \pi / L$ é o número de onda e $\omega=2 \pi / T$ é a frequência angular, sendo T o período da onda.

As ondas originadas em águas profundas viajam em direção à costa, ocorrendo variação do seu comprimento, velocidade e altura. Assume-se que o período da onda mantém-se inalterado, independendo da profundidade da água. Com os parâmetros da onda em águas profundas: $\mathrm{T}, \mathrm{L}_{0}$ e $\mathrm{H}_{0}$, o valor do comprimento da onda $\mathrm{L}$ e da altura da onda $\mathrm{H}$ em uma profundidade d são obtidos com as seguintes expressões:

$$
L=\frac{g T^{2}}{2 \pi} \tanh \left(2 \pi \frac{d}{L}\right) ; \quad H=H_{0}\left[\left\{1+\frac{4 \pi d / L}{\sinh (4 \pi d / L)}\right\} \tanh \left(2 \pi \frac{d}{L}\right)\right]^{-1 / 2} .
$$

onde a equação para L é obtida da equação de dispersão da onda linear, sendo $L_{0}=g T^{2} / 2 \pi$ e a equação para $\mathrm{H}$ é derivada da consideração de conservação de energia. $\mathrm{O}$ leito do mar levemente inclinado, em região de extensão limitada, é assumido horizontal, isto é, paralelo ao nível de água parada. Assim, para maciço inclinado a análise de estabilidade é feita localmente, para cada valor de $\mathrm{d}$.

A solução da onda linearizada de Stokes baseia-se na condição cinemática de superfície livre, em que não ocorre o fenômeno de rebentação da onda, o qual aparece quando a velocidade horizontal das partículas da crista da onda excede a velocidade da onda. Neste sentido, em águas rasas, uma condição adicional é imposta sobre as características da onda. A declividade da onda $\mathrm{H} / \mathrm{L}$ não pode aumentar além de certo valor crítico, no qual a onda quebra. Estudos (Horiwaka, 1978 apud Rahmah \& Jaber, 1986) apontam que as ondas possiveis com relação à condição de não quebra da onda são definidas pelo conjunto de parâmetros $(H, L, d)$ que satisfazem a condição:

$$
\mathrm{H}=\left\{(H, L, d), \quad \frac{H}{L} \leq \frac{1}{7} \tanh \left(\frac{2 \pi d}{L}\right)\right\} .
$$

Embora a teoria linearizada da onda assuma o leito do mar impermeável, pode-se mostrar que o uso desta teoria é justificável para leitos granulares, uma vez que as permeabilidades usuais dos sedimentos não afetam significativamente a sobrepressão induzida por tal onda (Dormieux et al., 1993). Além disso, esta teoria pode também ser aplicada como uma primeira aproximação no caso de leito levemente inclinado (Biesel, 1952 apud Dormieux \& Coussy, 1991).

\section{GEOMETRIA E CARREGAMENTO DO PROBLEMA}

O leito do mar é assumido como um espaço semi-infinito cujo topo, definido pelo plano $x_{2}=0$, pode estar inclinado um ângulo $\theta$ com relação à horizontal. Considerando-se 
condições de deformações planas, neste trabalho são analisados os casos de fundo do mar plano horizontal e fundo do mar plano inclinado, conforme Fig. 1.

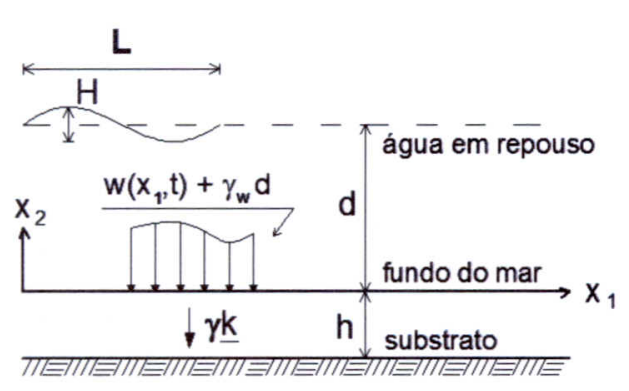

Fundo do mar plano horizontal $(\theta=0)$

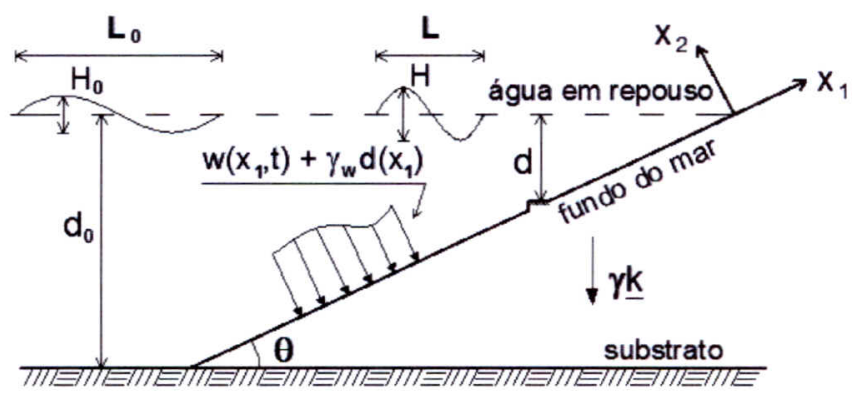

Fundo do mar plano horizontal $(\theta \neq 0)$

Figura 1 - Geometria do leito marinho submetido ao carregamento da onda e parâmetros da onda.

As forças de volume atuantes correspondem às forças de gravidade $\gamma \underline{k}=\rho g \underline{k}$ onde $g$ é o valor da aceleração da gravidade; $\rho=\rho^{w} \phi+(1-\phi) \rho^{s}$ representa a densidade mássica média do meio poroso; $\phi$ denota a porosidade que representa a fração do volume ocupado pelo fluido; $\rho^{w}$ e $\rho^{s}$ denotam as densidades mássicas da água e do sólido, respectivamente. Assume-se a ação da onda sobre o leito do mar modelada pela sobrepressão dada pela Eq. (1), com respeito ao valor hidrostático $\gamma_{w} d$. Constituem as forças de superfície $\underline{T}^{d}\left(x_{1}, t\right)$, atuantes sobre a fronteira $x_{2}=0$, a pressão hidrostática e a sobrepressão da onda:

$\underline{T}^{d}\left(x_{1}, t\right)=-\left(p_{0} \operatorname{sen} \psi+\gamma_{w} d\right) \underline{e}_{2} \quad\left(x_{2}=0\right)$.

onde $\psi=k x_{1}+\omega t ; d$ é um valor constante no caso do fundo do mar plano horizontal e $d=-x_{1} \operatorname{sen} \theta$ no caso do fundo do mar plano inclinado (veja Fig. 1).

Adota-se a hipótese de que a pressão do fluido é contínua através da interface em $x_{2}=0$. Usando $\delta u$ para denotar a diferença entre a poropressão no meio poroso com relação ao respectivo valor hidrostático, tem-se:

$$
\delta u\left(x_{1}, t\right)=p_{0} \operatorname{sen} \psi \quad\left(x_{2}=0\right) .
$$

A estabilidade, no sentido da teoria da análise limite, expressa a compatibilidade entre equilíbrio e resistência, isto é, a existência de um campo de tensões estaticamente admissível que obedece ao critério de resistência em todos os pontos:

$$
\begin{array}{cc}
\operatorname{div} \underline{\underline{\sigma}}+\gamma \underline{k}=0 & \left(x_{2} \leq 0\right) \\
\underline{\sigma} \cdot e_{2}=\underline{T}^{d} & \left(x_{2}=0\right) . \\
f(\underline{\underline{\sigma}}) \leq 0 & (\forall \underline{x})
\end{array}
$$

onde $\underline{\underline{\sigma}}$ é o tensor de tensões totais (sendo a tensão de tração adotada como positiva); $\underline{k}=-\left(\operatorname{sen} \theta \underline{e}_{1}+\cos \theta \underline{e}_{2}\right)$, sendo $\theta$ o ângulo de inclinação do fundo do mar. 


\section{ANÁLISE DA ESTABILIDADE DO MACIÇO CONSTITUIDO POR SOLO COESIVO}

Para características usuais de solos e onda, a velocidade da onda de água é pequena comparada com a velocidade de cisalhamento no solo. Isso sugere que os efeitos inerciais possam ser negligenciados (Mei \& Foda, 1981 apud Dormieux \& Coussy, 1991). Assim, a estabilidade do leito marinho sujeito a sobrepressão $p\left(x_{1}, t\right)$ durante um intervalo de tempo $\left[t_{0}, t_{1}\right]$ é equivalente à estabilidade em um tempo qualquer $t$ fixado dentro deste intervalo sob a sobrepressão $p\left(x_{1}\right)=p\left(x_{1}, t\right)$. Em outras palavras, a questão da estabilidade do leito oceânico pode ser tratada com base nos seguintes carregamentos: forças de gravidade, pressão hidrostática e uma sobrepressão estática definida pela função $p\left(x_{1}\right)=\operatorname{sen}\left(k x_{1}\right)$ (Dormieux \& Coussy, 1991).

O material que constitui o maciço é descrito pelo seu critério de resistência definido, nesta abordagem não-drenada, pelo critério de Tresca não-homogêneo com coesão $\mathrm{C}$ aumentando linearmente com a distância à fronteira $x_{2}=0$ :

$f\left(\underline{\underline{\sigma}}, x_{2}\right)=\max \left(\sigma_{i}-\sigma_{j}\right)-2 C\left(x_{2}\right) \leq 0 ; \quad C\left(x_{2}\right)=\eta_{c}\left|x_{2}\right|$.

onde $\sigma_{i}$ representa as tensões principais do tensor de tensões totais $\underline{\underline{\sigma}}$. O escalar $\eta_{c}$, chamado de gradiente de coesão, é o único parâmetro constitutivo requerido nesta análise. Este critério de resistência (critério linear isotrópico) é bem adaptado ao caso de argilas normalmente consolidadas, que constituem parte substancial do fundo oceânico.

\subsection{Abordagem estática}

A condição suficiente de estabilidade para o leito marinho constituído de material coesivo está vinculada à existência de um campo de tensões $\underline{\underline{\sigma}}$ estaticamente admissível com o carregamento definido por ( $\gamma, \gamma_{w}$ e $p_{0}$ ) e que seja compatível com o critério (7) em todos os pontos:

$$
\begin{aligned}
& \operatorname{div} \underline{\underline{\sigma}}+\gamma \underline{k}=0 \quad\left(x_{2} \leq 0\right) \\
& \underline{\underline{\sigma}} \cdot \underline{e}_{2}=-\left(p_{0} \operatorname{sen}\left(k x_{1}\right)+\gamma_{w} d\right) \cdot \underline{e}_{2} \quad\left(x_{2}=0\right) \text {. } \\
& \left(\left(\sigma_{11}-\sigma_{22}\right)^{2}+4 \sigma_{12}^{2}\right)^{1 / 2} \leq 2 \eta_{c}\left|x_{2}\right| \quad(\forall \underline{x}) .
\end{aligned}
$$

Um campo de tensão $\underline{\underline{\sigma}}$ estaticamente admissível com $\gamma, \gamma_{w}$ e $p_{0}$ deve ser construído. No caso em que $\theta$ é infinitamente pequeno, pode-se fazer uso dos resultados da teoria linear para modelagem do escoamento à montante da zona de rebentação e a análise de estabilidade é realizada localmente com parâmetros da onda correspondentes a cada valor de d (veja seção 2). Observa-se que, para o estudo do caso horizontal plano, a pressão hidrostática e as forças volumétricas de gravidade, por serem balanceadas por campos de tensão não-desviador, não contribuem para a ocorrência de estabilidade e somente a pressão induzida pela onda precisaria ser levada em conta. No entanto, os cálculos para análise da estabilidade serão apresentados para a situação mais geral, com $\theta \neq 0$ e utilizando-se $\theta=0$ obtêm-se as respostas relativas ao caso do fundo plano horizontal. 
Para determinação do campo tensorial que equilibra o carregamento da onda ${\underline{\underline{\sigma^{p}}}}^{p_{0}}$ utilizase uma solução particular de (8) obtida a partir da solução do problema clássico de Boussinesq, o qual estuda o efeito de uma carga linear vertical aplicada sobre a fronteira de um semi-espaço elástico (Dormieux \& Delage, 1988; Dormieux, 1989). O campo de tensão proposto é da forma:

$\sigma_{11}=-u-x_{2} \partial u / \partial x_{2} ; \quad \sigma_{22}=-u+x_{2} \partial u / \partial x_{2} ; \quad \sigma_{12}=x_{2} \partial u / \partial x_{1}$.

onde $u$ é a solução do problema:

$\left\{\begin{array}{ll}\Delta u=0 & \left(x_{2} \leq 0\right) \\ u\left(x_{1}, 0\right)=p\left(x_{1}\right) & \left(x_{2}=0\right)\end{array}\right.$.

onde $\Delta$ representa o operador Laplaciano. Desde que $p\left(x_{1}\right)=\operatorname{sen}\left(k x_{1}\right)$ seja uma função contínua e limitada, pode ser mostrado (Dormieux, 1989) que a seguinte integral define uma solução particular de (11):

$u\left(x_{1}, x_{2}\right)=\frac{1}{\pi} \int_{-\infty}^{+\infty} \operatorname{sen}\left(k x_{1}+\alpha k x_{2}\right) \frac{d \alpha}{1+\alpha^{2}}=\operatorname{sen}\left(k x_{1}\right) \mathrm{e}^{k x_{2}}$.

E o campo proposto fica da seguinte forma:

$\sigma_{11}^{p_{0}}=-p_{0} \operatorname{sen}\left(k x_{1}\right) \mathrm{e}^{k x_{2}}\left(1+k x_{2}\right)$

$\sigma_{22}^{p_{0}}=-p_{0} \operatorname{sen}\left(k x_{1}\right) \mathrm{e}^{k x_{2}}\left(1-k x_{2}\right)$.

$\sigma_{12}^{p_{0}}=p_{0} x_{2} k \cos \left(k x_{1}\right) \mathrm{e}^{k x_{2}}$

Os dois campos tensoriais $\underline{\underline{\sigma}}^{g}$ e $\underline{\underline{\sigma}}^{\text {hyd }}$, identificados abaixo por suas respectivas matrizes, equilibram as forças de gravidade e a pressão hidrostática aplicada sobre o fundo do mar (Dormieux, 1989):

$\underline{\underline{\sigma}}^{g}\left(x_{1}, x_{2}\right)=\not x_{2}\left(\begin{array}{cc}\cos \theta & \operatorname{sen} \theta \\ \operatorname{sen} \theta & \cos \theta\end{array}\right) ; \quad \underline{\sigma}^{\text {hyd }}\left(x_{1}, x_{2}\right)=\gamma_{w}\left(\begin{array}{cc}x_{1} \operatorname{sen} \theta & -x_{2} \operatorname{sen} \theta \\ -x_{2} \operatorname{sen} \theta & x_{1} \operatorname{sen} \theta\end{array}\right)$.

Assim, $\underline{\underline{\sigma}}=\underline{\underline{\sigma}}^{g}+\underline{\underline{\sigma}}^{h y d}+\underline{\underline{\sigma}}^{p_{0}}$ é solução de (8). A fim de ser compatível com o critério de resistência do material, este campo deve ainda satisfazer a condição (9). Com a aplicação das condições mais restritivas para $x_{2}$ e $x_{1}$, a condição suficiente de estabilidade fica definida por $p_{0} \leq\left(\eta_{c}-\gamma^{\prime} \operatorname{sen} \theta\right) / k$. Assim, o teorema do limite inferior afirma que a carga limite deve obedecer a seguinte condição:

$p_{0 \text { coes }}^{\lim } \geq \frac{\eta_{c}-\gamma^{\prime} \operatorname{sen} \theta}{k}$.

onde $\gamma^{\prime}=\gamma-\gamma_{w}$ é o peso específico submerso do solo. Observa-se que se $\eta_{c}<\gamma^{\prime} \operatorname{sen} \theta$, a condição acima não gera informações adicionais para o problema. Por outro lado, a inequação $\eta_{c} \geq \gamma^{\prime} \operatorname{sen} \theta$ pode ser interpretada como uma condição suficiente de estabilidade do leito marinho sob a ação combinada das forças de gravidade e pressão hidrostática. Verifica-se ainda que no caso de um fundo do mar horizontal, isto é com $\theta=0$, o limite inferior da carga limite não depende de $\gamma^{\prime}$. E, finalmente, comparando o caso mais geral, de fundo plano

CILAMCE 2015

Proceedings of the XXXVI Iberian Latin-American Congress on Computational Methods in Engineering

Ney Augusto Dumont (Editor), ABMEC, Rio de Janeiro, RJ, Brazil, November 22-25, 2015 
inclinado, ao caso do fundo plano horizontal, o efeito da inclinação é equivalente a uma redução do gradiente de coesão no valor de $\gamma^{\prime} \operatorname{sen} \theta$.

\subsection{Abordagem cinemática}

Para um dado ponto $\mathrm{M}$ sobre o leito do mar, em $x_{2}=0$, os campos de velocidade $\underline{V}^{\alpha l}$ definidos na Fig. 2 são considerados:

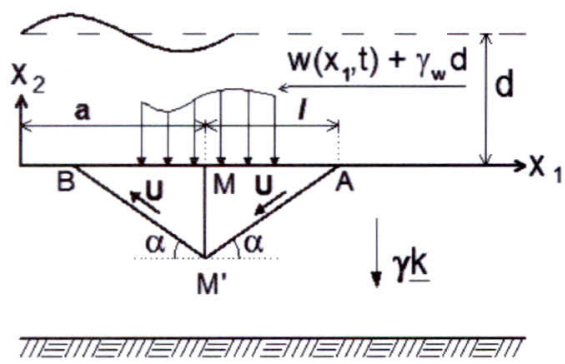

Fundo do mar plano horizontal $(\theta=0)$

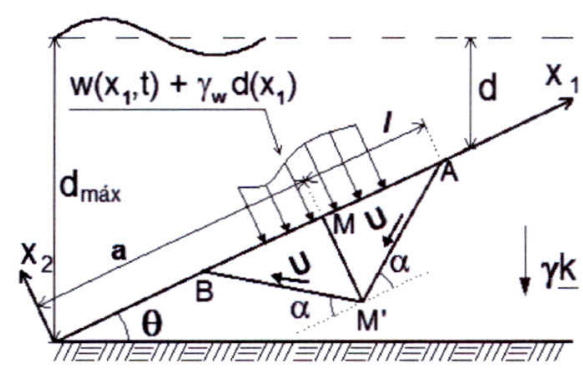

Fundo do mar plano horizontal $(\theta \neq 0)$

Figura 2- Campo de velocidade de corpo rígido $\underline{V}^{\alpha l}$.

Os dois blocos triangulares $M M^{\prime} A$ e $M M^{\prime} B$ mantêm-se indeformados e transladam paralelamente a $M^{\prime} A$ e $M ' B$ :

$\underline{V}^{\alpha l}\left(M^{\prime} M A\right)=U\left(-\cos \alpha \underline{e_{1}}-\operatorname{sen} \alpha \underline{e_{2}}\right)$

$\underline{V}^{\alpha l}\left(M^{\prime} M B\right)=U\left(-\cos \alpha \underline{e_{1}}+\operatorname{sen} \alpha \underline{e_{2}}\right)$.

onde U é um escalar positivo. Em qualquer outro lugar na massa de solo, o campo de velocidade permanece igual a zero. As características geométricas do campo de velocidade são a abcissa $a$ do ponto $\mathrm{M}$, o comprimento $l=M A=M B>0$, e o ângulo $\alpha$ que deve satisfazer a condição $\alpha \in] 0, \pi / 2\left[\right.$. Os segmentos $M^{\prime} A$ e $M^{\prime} B$ constituem as linhas de descontinuidade deste campo de velocidades. A velocidade de descontinuidade $\underline{W}$ entre os blocos $M M^{\prime} A$ e $M M^{\prime} B$ é paralela a $M^{\prime} M$ e vale $\underline{W}=2 U$ sen $\alpha e_{2}$.

A potência resistente máxima consiste na soma das contribuições relacionadas a cada linha de descontinuidade do campo de velocidade, isto é, $M^{\prime} A, M^{\prime} M$ e $M^{\prime} B$ :

$P_{r m}\left(V^{\alpha l}\right)=U\left\{\int_{M^{\prime} A} C\left(x_{2}\right) d s+\int_{M^{\prime} B} C\left(x_{2}\right) d s+2 \operatorname{sen} \alpha \int_{M^{\prime} M} C\left(x_{2}\right) d s\right\}$.

onde $C\left(x_{2}\right)$ é a coesão, conforme critério de resistência de Tresca dado em (7). Nota-se que $P_{r m}\left(\underline{V}^{\alpha l}\right)$ depende apenas do critério de resistência e do campo de velocidade escolhido. Esta quantidade é independente do carregamento. Usando a definição de $C\left(x_{2}\right)$, obtém-se:

$P_{r m}\left(V^{\alpha l}\right)=\eta_{c} l^{2} U \frac{\operatorname{sen} \alpha\left(1+\operatorname{sen}^{2} \alpha\right)}{\cos ^{2} \alpha}$.

Para o cálculo da potência externa considerar-se-á novamente a situação mais geral, com, $\operatorname{com} \theta \neq 0$. Utilizando-se $\theta=0$ obtêm-se as respostas relativas ao fundo plano horizontal. A potência externa consiste na soma das contribuições relacionadas às forças de gravidade $P_{g}$, 
pressão hidrostática $P_{\text {hyd }}$ e pressão da onda $P_{p_{0}}$. Assim, o teorema do limite superior expressa que a inequação:

$$
P_{p_{0}}\left(\underline{V}^{\alpha l}\right)+P_{h y d}\left(\underline{V}^{\alpha t}\right)+P_{g}\left(\underline{V}^{\alpha t}\right) \leq P_{r m}\left(\underline{V}^{\alpha l}\right) \text {. }
$$

é uma condição necessária para estabilidade do fundo do mar plano inclinado. A potência externa relacionada à pressão da onda é dada por:

$$
P_{p_{0}}=\int_{-\infty}^{+\infty} \underline{V}^{\alpha l}\left(x_{1}, 0\right) \cdot\left(-p_{0} p\left(x_{1}\right) \underline{e_{2}}\right) d x_{1}=\frac{2 U p_{0}}{k} \operatorname{sen} \alpha \cos k a(1-\cos k l) \text {. }
$$

onde "." representa o produto escalar.

As potências externas relacionadas à pressão hidrostática $P_{h y d}$ e às forças de gravidade $P_{g}$ são dadas por:

$$
\begin{aligned}
& P_{h y d}=\int_{-\infty}^{+\infty} \underline{V} \underline{V}^{\alpha t}\left(x_{1}, 0\right) \cdot\left(-\gamma_{w} d\left(x_{1}\right) \underline{e_{2}}\right) d x_{1}=-U \gamma_{w} l^{2} \operatorname{sen} \alpha \operatorname{sen} \theta . \\
& P_{g}=-\int_{\Omega} \underline{V^{\alpha l}}(M) \cdot \gamma\left(\underline{e_{1}} \operatorname{sen} \theta+\underline{e_{2}} \cos \theta\right) d \Omega=U \gamma l^{2} \operatorname{sen} \alpha \operatorname{sen} \theta .
\end{aligned}
$$

onde $d\left(x_{1}\right)=d_{\max }-x_{1} \operatorname{sen} \theta$. Observa-se que $P_{h y d}$ e $P_{g}$ são dados pela mesma expressão, mas com sinais opostos, representando, respectivamente, forças desestabilizadoras e forças de resistência. Substituindo (18), (20), (21) e (22) em (19) e aplicando as condições mais restritivas para $\alpha, l$ e $a$ obtém-se a condição necessária para estabilidade do fundo do mar plano inclinado: $p_{0} \leq\left(\eta_{c}-\gamma^{\prime} \operatorname{sen} \theta\right) / k$, onde $\gamma^{\prime}=\gamma-\gamma_{w}$ é o peso específico submerso do solo. Assim, o teorema do limite superior afirma que a carga limite deve obedecer a seguinte condição:

$$
p_{0 \text { coes }}^{\lim } \leq \frac{\eta_{c}-\gamma^{\prime} \operatorname{sen} \theta}{k} .
$$

onde o gradiente de coesão $\eta_{c}$ e o número de onda $k$ são grandezas positivas. No caso de um fundo do mar horizontal, isto é com $\theta=0$, o limite inferior da carga limite não depende de $\gamma^{\prime}$. Observa-se que para ambos os casos: fundo do mar plano horizontal e inclinado as condições mais restritivas da inequação (19), isto é, o menor valor da potência resistente e o maior valor da potência externa, ocorrem em $\alpha \rightarrow 0, l \rightarrow 0$ e $a \rightarrow 0$. Isto é, o melhor mecanismo da classe estudada é instável quando a zona deformada tende ao ponto $\mathrm{M}$ (veja Fig. 2). Essa propriedade do mecanismo ótimo deve ser atribuída à condição $C\left(x_{2}=0\right)=0$ (Dormieux, 1989).

\subsection{Delimitação da carga limite}

As expressões (15) e (23) fornecem um limite inferior e superior, respectivamente, da amplitude da carga limite da onda $p_{0}^{\text {lim }}$, modelada conforme a teoria linear, para o caso mais abrangente de fundo do mar plano inclinado. Os valores definidos por esses limites devem também obedecer à condição de validade do modelo linear, dada pelo contorno $\mathrm{H}$ conforme fórmula (3). Substituindo-se a expressão de $p_{0}$ em termos dos parâmetros da onda $\mathrm{H}, \mathrm{L}$ e d, 
conforme Eq. (1), nas expressões para $p_{0}^{\lim }$ citadas acima é possível traçar curvas em função dos valores limites $\mathrm{H} / \mathrm{L}$ e $\mathrm{d} / \mathrm{L}$. Verifica-se que as curvas estabelecidas pelo limite inferior (15) e pelo limite superior (23) se superpõem, conforme pode ser visto na Fig.3 para os casos de fundo plano horizontal $(\theta=0)$ e inclinado, para dois valores do ângulo $\theta$. Nessas figuras está também ilustrado o domínio de validade do modelo da onda linear.

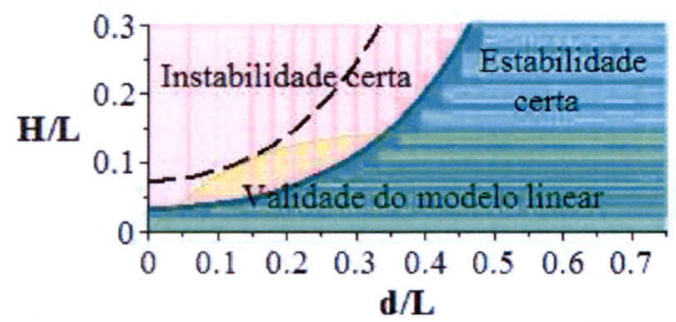

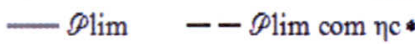

(a)

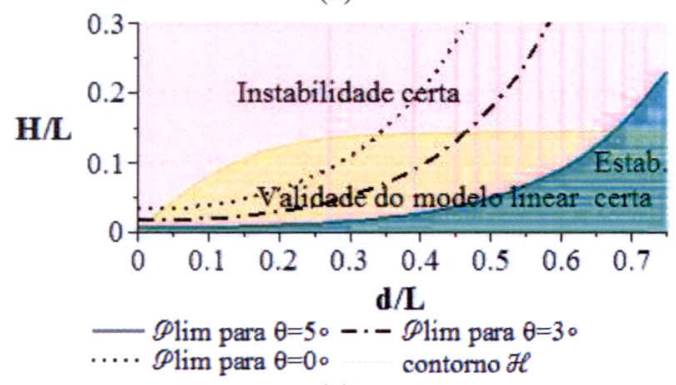

(c)

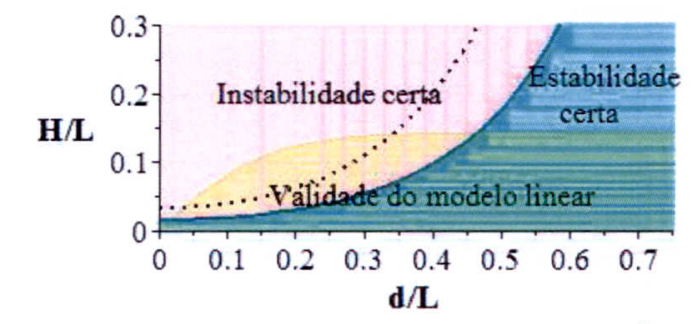

- Plim para $\theta=3 \circ \cdots \cdots \mathscr{P l i m}$ para $\theta=0$ 。 contorno $\mathscr{H}$

(b)

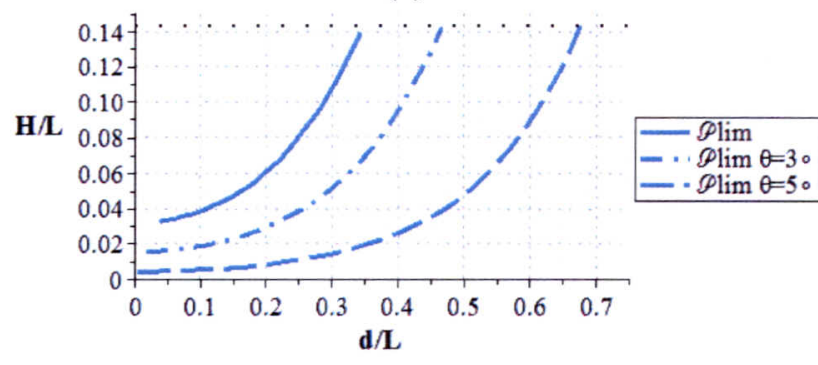

(d)

Figura 3 - Carga limite $p_{0}^{\lim }=p^{\lim }$ traçada em função da combinação dos parâmetros da onda [H/L, d/L], $\operatorname{com} \eta_{c}=0,1 \gamma_{w}$, para: (a) fundo do mar horizontal; (b) fundo do mar inclinado a $\theta=3^{\circ}$ e (c) fundo do mar inclinado a $\theta=5^{\circ}$; (d) valor da carga limite que satisfaz também a condição de validade do modelo linear.

Assim, pode-se afirmar que a teoria da análise limite aplicada ao problema de estabilidade do leito do mar constituído por material de Tresca e submetido ao carregamento da onda, modelada conforme teoria linear de Stokes, fornece o valor exato da amplitude segura máxima, $p_{0}^{\text {lim }}$. No caso do fundo do mar horizontal, observa-se que para o valor crítico $\eta_{c}^{*} \approx 0,225 \gamma_{w}$ de $\eta_{c}$, o contorno convexo de $p_{0 A C}^{\text {lim }}$ e o contorno côncavo de $\mathrm{H}$ são tangentes e o domínio de cargas que obedece simultaneamente à condição de não quebra de onda e instabilidade certa do fundo do mar reduz-se ao conjunto vazio (veja na Fig. 3 (a) o traçado de $p_{0}^{\lim } \operatorname{com} \eta_{c}^{*} \approx 0,225 \gamma_{w}$ ). Em outras palavras, para coeficiente de coesão maior ou igual a $\eta_{c}{ }^{*}$, não existe uma onda fisicamente possível capaz de causar instabilidade em um fundo do mar horizontal, quando a onda linear de Stokes é utilizada. Na medida em que o valor de $\eta_{c}$ diminui, a partir de $\eta_{c}=\eta_{c}{ }^{*}$, o domínio de cargas possíveis que assegura a estabilidade diminui e, logo, o domínio de cargas possíveis que causam instabilidade aumenta.

\section{ANÁliSE DA ESTABILIDADE DO MACIÇO CONSTITUIDO POR SOLO GRANULAR}

Para materiais granulares, a resistência do meio depende explicitamente do valor da poropressão, isto é, $f(\underline{\underline{\sigma}}(\underline{x}))=f(\underline{\underline{\sigma}}(\underline{x})+u(\underline{x}) \underline{\underline{\underline{x}}})$. Sendo descrita classicamente pelo critério de Coulomb sem coesão: 
$f \underline{\underline{\sigma}}+u \underline{1})=\max _{i, j}\left(\left(\sigma_{i}+u\right)(1+\operatorname{sen} \varphi)-\left(\sigma_{j}+u\right)(1-\operatorname{sen} \varphi)\right) \leq 0$.

onde $\varphi$ é o ângulo de atrito do material. Assim, torna-se necessário conhecer a distribuição de poropressões no maciço.

Introduz-se a mudança de variáveis $\underline{\underline{\sigma^{\prime}}}=\underline{\underline{\sigma}}+u \underline{\underline{1}}$, onde $\underline{\underline{\sigma^{\prime}}}$ é o tensor de tensões efetivas (sendo a tensão de tração adotada como positiva) e $u$ é a poropressão total, a qual é definida pela soma de um valor inicial $u_{\text {inic }}$ e de uma variação $\delta u$. Usando esta mudança de variáveis, a força atuante sobre o leito do mar (4) e introduzindo $\gamma^{\prime}=\gamma-\gamma_{w}$, a condição de estabilidade (6) fica da seguinte forma:

$$
\begin{array}{cc}
\operatorname{div} \underline{\underline{\sigma^{\prime}}}-\underline{\operatorname{grad}} \delta u+\gamma^{\prime} \underline{k}=0 & \left(x_{2} \leq 0\right) \\
\underline{\sigma^{\prime} \cdot e_{2}}=0 & \left(x_{2}=0\right) \\
f \underline{\left.\underline{\sigma^{\prime}}(\underline{x})\right) \leq 0} & (\forall \underline{x})
\end{array} .
$$

A vantagem da mudança de variável é que permite escrever o critério de resistência em termos de tensões efetivas:

$$
f\left(\underline{\underline{\sigma^{\prime}}}\right)=\max _{i, j}\left(\sigma_{i}^{\prime}(1+\operatorname{sen} \varphi)-\sigma_{j}^{\prime}(1-\operatorname{sen} \varphi)\right) \leq 0 .
$$

Entretanto, a mudança de variável usada implica também uma mudança no valor das forças envolvidas. Introduzindo-se o vetor adimensional gradiente hidráulico:

$$
\underline{i}=-\frac{1}{\gamma_{w}} \underline{\operatorname{grad}} \delta u \text {. }
$$

o carregamento em tensões efetivas fica definido pela soma de duas densidades de força de corpo: a densidade $\gamma^{\prime} \underline{k}$, definida pelo peso unitário submerso $\gamma^{\prime}=\gamma-\gamma_{w}$ e a densidade $\gamma_{w} \underline{i}$, relacionada às forças do fluxo de percolação, as quais são paralelas ao vetor de fluxo relativo de massa fluida $\underline{w}$, de acordo com a lei de Darcy.

\subsection{Transporte da massa fluida no meio poroso}

Considerando a hipótese das transformações infinitesimais, a equação da conservação de massa do meio poroso pode ser expressa por (Coussy, 2004):

$\dot{m}+\operatorname{div} \underline{w}=0$.

onde $m=\rho^{w} \phi$ é o conteúdo de massa fluida Lagrangeano, sendo $\phi$ a porosidade Lagrangeana e $\underline{w}(\underline{x}, t)$ é o vetor de fluxo relativo de massa fluida Euleriano dado por $\underline{w}=\rho^{w} \underline{q}$, onde $\underline{q}$ é o vetor de filtração.

A difusão da massa fluida através do meio poroso é classicamente descrita pela Lei de Darcy, que relaciona $\underline{w}(\underline{x}, t)$ à poropressão $u$ do fluido. No caso isotrópico e negligenciando termos inerciais tem-se:

$\underline{q}=k \underline{\underline{1}} \cdot\left(-\underline{\operatorname{grad}} u+\rho^{w} \underline{g}\right)$.

onde $k$ é a constante de permeabilidade. 
Adicionalmente, adota-se a hipótese de que as duas fases que constituem o meio poroso são incompressíveis. Essa propriedade da matriz sólida implica que as deformações volumétricas do esqueleto sejam relacionadas diretamente com a variação de porosidade:

$m / \rho^{w}-\phi_{0}=\operatorname{tr} \underline{\underline{\varepsilon}}$.

onde $\phi_{0}$ é a porosidade inicial e $\underline{\underline{\varepsilon}}$ é o tensor de deformações linearizado. A equação acima expressa o princípio de que nenhuma quantidade de massa fluida pode ser adicionada a menos que exista uma deformação volumétrica do esqueleto correspondente. Finalmente, a equação de difusão que relaciona as deformações do esqueleto e poropressões é deduzida das equações (28), (29) e (30):

$\operatorname{tr} \underset{\underline{\varepsilon}}{=}=k \Delta u$.

A equação (31) introduz um acoplamento entre o fluido e o esqueleto que torna impossível o cálculo separado da poropressão. De uma maneira geral, a resolução numérica de um problema de valor limite no domínio das solicitações cíclicas apresenta uma grande complexidade. Encontram-se dificuldades de ordem reológica e relacionadas ao tratamento numérico do acoplamento fluido-esqueleto. Neste sentido, destaca-se a importância das pesquisas experimentais realizadas para compreensão do papel da água intersticial no comportamento de solos sob carregamento cíclico. O volume de dados experimentais sobre o comportamento de areias submetidas a uma solicitação cíclica em condição drenada é relativamente raro. $\mathrm{O}$ fato dos ensaios não-drenados terem sido privilegiados deve-se provavelmente a importância do fenômeno de liquefação, em função da ameaça devido a sua dimensão catastrófica. Uma relação entre os dois tipos de ensaio foi apresentado em Dormieux (1989).

\subsection{Formulação baseada no método desacoplado}

Neste trabalho, considera-se uma simplificação que consiste em desacoplar artificialmente o cálculo da pressão intersticial, segundo uma ideia introduzida por Seed et al. (1976), a qual baseia-se sobre a partição das deformações em contribuições reversíveis e irreversíveis: $\operatorname{tr} \underline{\underline{\varepsilon}}=\operatorname{tr} \underline{\underline{\varepsilon}}^{\text {rev }}+\operatorname{tr} \underline{\underline{\varepsilon}}^{i r r}$ (Dormieux, 1989). De acordo com esta hipótese, as forças de percolação são também particionadas em contribuições reversível e irreversível: $\gamma_{w} \underline{\underline{i}}^{r e v}=-\underline{\operatorname{grad}} \delta u^{\text {rev }}$ e $\gamma_{w} \underline{i}^{i r r}=-\underline{\operatorname{grad}} \delta u^{i r r}$, onde as notações $\delta u^{\text {rev }}$ e $\delta u^{i r r}$ representam os campos de poropressões relacionados à solução do problema com deformações volumétricas reversíveis e irreversíveis, respectivamente. A ideia de desacoplamento conduz a definição de dois problemas: o reversível, que se refere ao problema poroelástico com carregamento definido pela função de pressões e o irreversível, que se refere a um problema cujo carregamento é definido pelo campo de deformações irreversíveis $\underline{\underline{\varepsilon}}^{i r r}(\underline{M}, t)$, considerado como dado. As condições iniciais dos dois problemas são consideradas nulas.

Contribuição reversível das forças de percolação. Considera-se o esqueleto do solo homogêneo, isotrópico e elástico linear; a água contida nos poros é incompressível e a permeabilidade do solo saturado é considerada isotrópica. Assume-se ainda que em um ponto M localizado a uma distância infinita da origem do sistema, deformações, poropressões e deslocamentos são nulos. 
Para resolver o problema elástico em tensões efetivas Dormieux \& Delage (1988) apresentou uma solução obtida por meio da resolução de dois problemas associados. Do primeiro problema associado obtêm-se as componentes do tensor de tensão total, através da integração da solução elementar de Boussinesq, com $v=1 / 2$. Do segundo problema associado, representado pelo problema de Dirichlet obtém-se a poropressão da água. Nessas condições, os autores mostram que no caso de um meio poroso composto por esqueleto homogêneo, isotrópico e elástico linear, nenhuma deformação volumétrica ocorre durante o carregamento da onda. Isso significa que não existe acoplamento entre a água do poro e o esqueleto. Considerando o carregamento da onda de acordo com a teoria linearizada, a distribuição de poropressões e as componentes do tensor de tensões efetivas ficam da seguinte forma:

$$
\begin{aligned}
& \delta u^{r e v}\left(x_{1}, x_{2}, t\right)=p_{0} \operatorname{sen} \psi \mathrm{e}^{k x_{2}}, \quad \psi=k x_{1}+\omega t . \\
& \sigma_{11}^{\prime}=-p_{0} x_{2} k \operatorname{sen} \psi \mathrm{e}^{k x_{2}} ; \quad \sigma_{12}^{\prime}=p_{0} x_{2} k \cos \psi \mathrm{e}^{k x_{2}} ; \quad \sigma_{22}^{\prime}=p_{0} x_{2} k \operatorname{sen} \psi \mathrm{e}^{k x_{2}} .
\end{aligned}
$$

É importante notar que as tensões totais são as mesmas no esqueleto poroso assim como no material monofásico elástico linear isotrópico homogêneo com $v=1 / 2$ (isto é, incompressível).

Contribuição irreversível das forças de percolação. Para um carregamento puramente desviador cujo ciclo elementar seja "pouco intenso", considera-se que a medida da pressão intersticial em condição não-drenada fornece diretamente o valor das deformações irreversíveis (em condição drenada) (Dormieux, 1989). Com essa hipótese, pode-se admitir que as condições de drenagem afetam de maneira negligenciável o valor das deformações encontradas. Em particular, o resultado correspondente ao ensaio não-drenado $\Delta \varepsilon_{n d}$ pode servir de aproximação para outros tipos de ensaio, isto é, $\Delta \varepsilon_{v}^{i r r}=\Delta \varepsilon_{n d}$. A quantidade $\Delta \varepsilon_{n d}$ é vinculada a geração de sobrepressão intersticial $\Delta u$ pela relação:

$\Delta u=-K_{v} \Delta \varepsilon_{n d}$.

onde $K_{v}$ é o módulo de compressibilidade volumétrica.

Limita-se ao caso em que as deformações irreversíveis são do tipo uniaxial e correspondem a uma densificação do tipo "oedométrica" segundo a direção $\underline{e}_{2}: \underline{\underline{\varepsilon}}^{i r r}\left(x_{2}, t\right)=C\left(x_{2}, t\right) \underline{e_{2}} \otimes \underline{e_{2}}$.

Neste estudo é utilizado um modelo simples (Seed et al., 1976; Rahman \& Jaber, 1986) no qual a variação da poropressão durante um passo de tempo é dada por dois processos simultâneos: (I) geração de poropressão causada pela ação cíclica das tensões cisalhantes (informação obtida de ensaios não-drenados); e (II) dissipação de poropressão causada pelo fluxo. Considerando (31) e (34), o problema fica definido pela equação de difusão nãohomogênea e condições de contorno:

$$
\begin{aligned}
& c_{v} \frac{\partial^{2} \delta u^{i r r}}{\partial x_{2}{ }^{2}}=\frac{\partial}{\partial t} \delta u^{i r r}+f, \quad f=\frac{\gamma^{\prime}}{T} c x_{2} e^{r x_{2}} . \\
& \delta u^{i r r}(0, t)=0 ; \quad \frac{\partial \delta u^{i r r}}{\partial x_{2}}(-h, t)=0 ; \quad \delta u^{i r r}\left(x_{2}, 0\right)=0 .
\end{aligned}
$$


onde $c_{v}=k /\left(\gamma_{w} m_{v}\right)$ é o coeficiente de consolidação, com $m_{v}$ o coeficiente de variação volumétrica; h é a espessura da camada de solo e $f$ é o termo fonte apresentado em Rahman \& Jaber (1986): c é um coeficiente que depende dos parâmetros da onda, densidade relativa do solo $D_{r}$ e parâmetros do modelo; $r$ é um coeficiente que depende do carregamento da onda e parâmetros do modelo.

A Fig. 4 apresenta os perfis da pressão intersticial "irreversível" obtidos no maciço, conforme o conjunto de parâmetros apresentados na Tabela 1, resultantes de diferentes ciclos de carregamentos, onde $n=t / T$. A solução do problema (35-36) foi obtida com auxílio do software simbólico Maple.

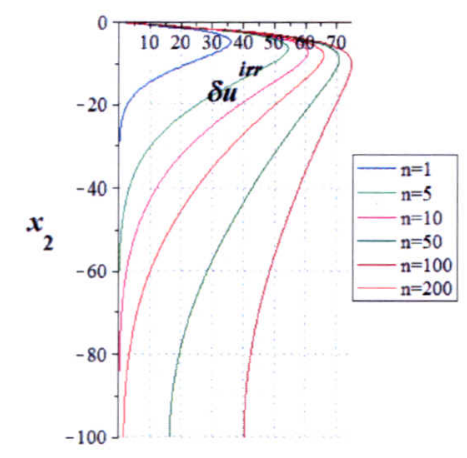

Figura 4 - Perfis de poropressão de origem anelástica em [kPa] no interior do maciço, de acordo com o número de ciclos " $n$ ” do carregamento.

Tabela 1. Parâmetros de cálculo.

\begin{tabular}{llll}
\hline \multicolumn{1}{c}{ Solo } & \multicolumn{1}{c}{ Solicitação } & \multicolumn{1}{c}{ Geometria } & Modelo \\
\hline$m_{v}=3,13 \cdot 10^{-5} m^{2} / k N ; \quad D_{r}=0,7$ & $T_{\text {onda }}=11,3 s$ & $d=14 m$ & $\bar{a}=0,287$ \\
$k=10^{-3} \mathrm{~m} / \mathrm{s} ; \quad \gamma^{\prime} / \gamma_{w}=0,77$ & $H_{0} / L_{0}=0,055$ & $h=100 m$ & $\bar{b}=0,095$ \\
\hline
\end{tabular}

Na Fig. 4 observa-se uma acumulação progressiva da pressão em função do tempo, isto é, em um ponto, a pressão intersticial cresce em função do número de ciclos. A nulidade da pressão na superfície superior do maciço é imposta pela condição de drenagem. O perfil é marcado pela presença de um máximo em um $x_{2}^{*}$, situado a uma pequena distância da superfície superior do maciço. Para $x_{2}<x_{2}^{*}$, as forças de fluxo associadas são orientadas para a base da camada de solo, agindo de forma estabilizadora, no sentido em que são equivalentes a um aumento do peso volumétrico submerso do solo. Ao contrário, para $x_{2}>x_{2}^{*}$, elas são orientadas para a superfície superior do maciço, agindo de forma instabilizadora, pois diminuem o peso volumétrico aparente. A forma do perfil obtido anuncia que um risco de instabilidade superficial pode acompanhar o processo de acumulação de pressão. Observa-se que a derivada em relação à coordenada $x_{2}$ é uma quantidade negativa e decrescente para $x_{2} \in\left[x_{2}^{*}, 0\right]$ e positiva para $x_{2} \leq x_{2}^{*}$. O valor do gradiente da pressão intersticial na superfície constitui um parâmetro essencial do perfil do ponto de vista do cálculo de estabilidade, como será visto a diante. Ele representa, em valor absoluto, o valor da força de fluxo de origem anelástica no topo do maciço. Nota-se:

$\gamma_{w} i^{i r r}(0)=-\left.\frac{\partial}{\partial x_{2}} \delta u^{i r r}\right|_{x_{2}=0}$ 


\subsection{Abordagem estática}

A condição suficiente de estabilidade para o leito marinho constituído de material granular está vinculada à existência de um campo de tensões efetivas estaticamente admissível com o carregamento definido pelas densidades de força de corpo ( $\gamma^{\prime} \underline{k}$ e $\left.\gamma_{w} \underline{i}\right)$ e que seja compatível com o critério (26) em todos os pontos:

$$
\begin{array}{cc}
\operatorname{div} \underline{\underline{\sigma^{\prime}}+\gamma_{w} \underline{i}+\gamma^{\prime} \underline{k}=0} & \left(x_{2} \leq 0\right) \\
\underline{\sigma}^{\prime} \cdot \underline{e_{2}}=0 & \left(x_{2}=0\right) \\
\left(\left(\sigma_{11}^{\prime}-\sigma_{22}^{\prime}\right)^{2}+4{\sigma_{12}^{\prime}}^{2}\right)^{1 / 2} \leq-\left(\sigma_{11}^{\prime}+\sigma_{22}^{\prime}\right) \operatorname{sen} \varphi & (\forall \underline{x}) .
\end{array}
$$

onde o ângulo de atrito $\varphi$ é suposto inferior a $\pi / 4$.

Observa-se que para este critério de resistência, campos de tensão não-desviadores contribuem para a estabilidade, ao contrário do de Tresca utilizado anteriormente. E os carregamentos considerados nas análises para fundo plano horizontal e inclinado são iguais. Assim, os cálculos para análise da estabilidade do maciço constituído por solo granular serão apresentados para a situação mais geral, $\operatorname{com} \theta \neq 0$. Utilizando-se $\theta=0$ obtêm-se respostas relativas ao fundo plano horizontal.

Para o cálculo das forças relacionadas ao fluxo de percolação, considera-se a distribuição de poropressão relacionada às contribuições reversível (32) e irreversível, ou seja, $\underline{i}^{=} \underline{i}^{e}+\underline{i}^{i r r}$ :

$$
\begin{aligned}
& \gamma_{w} \underline{i}^{e}=-p_{0} k \mathrm{e}^{k x_{2}}\left(\cos \psi \underline{e_{1}}+\operatorname{sen} \psi \underline{e_{2}}\right), \quad \psi=k x_{1}+\omega t . \\
& \gamma_{w} \underline{i}^{i r r}=\gamma_{w} i^{i r r}\left(x_{2}\right) \underline{e_{2}} .
\end{aligned}
$$

onde $\underline{i}^{i r r}$ é uma função independente de $x_{1}$, paralelo a $\underline{e}_{2}$ cuja amplitude é determinada pela resolução do problema de difusão (35-36). Variações de $\underline{i}^{\text {irr }}$ sobre um período são negligenciáveis diante daquelas de $\underline{i}^{e}$ e o argumento t poderá ser omitido no estudo de estabilidade no decurso de um ciclo de carregamento. Os cálculos numéricos apresentados na seção anterior conduzem a limitar o estudo aos carregamentos para os quais a função $\underline{i}^{i r r}$ possui as seguintes propriedades-(Dormieux, 1989):

(I) existe um real $x_{2} *<0$ tal que: $\left(\forall x_{2}<x_{2} *\right) \quad \underline{i}^{i r r}\left(x_{2}\right) \leq 0 ;\left(\forall x_{2} \geq x_{2} *\right) \quad \underline{i}^{i r r}\left(x_{2}\right) \geq 0$;

(II) $\left(\forall x_{2} \leq 0\right) \quad \int_{x_{2}}^{0} i^{i r r}(\alpha) d \alpha \geq 0$.

Introduzem-se os campos de tensões efetivas $\underline{\underline{\sigma}}_{1}^{\prime}, \underline{\underline{\sigma}}_{2}{ }_{2}$ e $\underline{\underline{\sigma}}_{3}^{\prime}$ que equilibram, respectivamente, as componentes de densidade volumétrica das forças externas $\gamma^{\prime} \underline{k}, \gamma_{w} \underline{i}$ e $\gamma_{w} \underline{i}^{i r r}$. Eles são definidos pelas seguintes matrizes na base $\left(\underline{e}_{1}, \underline{e}_{2}\right)$ :

$\underline{\underline{\sigma}}_{1}^{\prime}=\gamma^{\prime} x_{2}\left(\begin{array}{cc}K_{1}\left(x_{2}\right) & \operatorname{sen} \theta \\ \operatorname{sen} \theta & \cos \theta\end{array}\right), \quad \underline{\underline{\sigma}}_{2}^{\prime}=x_{2} A\left(x_{2}\right)\left(\begin{array}{cc}-\operatorname{sen} \psi & \cos \psi \\ \cos \psi & \operatorname{sen} \psi\end{array}\right), \quad \underline{\underline{\sigma}}_{3}^{\prime}=\gamma^{\prime} x_{2}\left(\begin{array}{cc}K_{2}\left(x_{2}\right) & 0 \\ 0 & Q\left(x_{2}\right)\end{array}\right)$,

$\operatorname{com} \quad A\left(x_{2}\right)=k p_{o} \mathrm{e}^{k x_{2}} ; \quad \psi=k x_{1}-\omega t ; \quad Q\left(x_{2}\right)=\frac{\gamma_{w}}{\gamma^{\prime}} \frac{1}{x_{2}} \int_{x_{2}}^{0} i^{i r r}(\alpha) d \alpha$ 
Sendo a escolha de $K_{1}\left(x_{2}\right)$ e $K_{2}\left(x_{2}\right)$ arbitrária. Superpondo esses três campos, obtém-se um campo de tensões $\underline{\underline{\sigma^{\prime}}}$ que equilibra as forças externas, e cujas componentes são:

$\sigma_{11}^{\prime}=\gamma^{\prime} x_{2}\left(K-\frac{A}{\gamma^{\prime}} \operatorname{sen} \psi\right), \quad \sigma_{22}^{\prime}=\gamma^{\prime} x_{2}\left(X+\frac{A}{\gamma^{\prime}} \operatorname{sen} \psi\right), \quad \sigma_{12}^{\prime}=\gamma^{\prime} x_{2}\left(\operatorname{sen} \theta+\frac{A}{\gamma^{\prime}} \cos \psi\right)$

$\operatorname{com} \quad K=K_{1}+K_{2} \quad ; \quad X=\cos \theta+Q$

onde o argumento $x_{2}$ foi omitido.

O problema consiste em encontrar a que condição sobre a função $i^{i r r}\left(x_{2}\right)$, um campo de tensões do tipo $\underline{\underline{\sigma}}^{\prime}$ compatível em todo ponto do maciço com a capacidade de resistência definida por $\varphi$ pode ser exibido. Substituindo as componentes de $\underline{\underline{\sigma}}^{\prime}$ em (39) verifica-se que a construção deste campo baseia-se na existência de uma função $K$ que satisfaça:

$\left(\left(K-X-\frac{2 A}{\gamma^{\prime}} \operatorname{sen} \psi\right)^{2}+4\left(\operatorname{sen} \theta+\frac{A}{\gamma^{\prime}} \cos \psi\right)^{2}\right)^{1 / 2} \leq(K+X) \operatorname{sen} \varphi, \quad\left(\forall x_{2} \leq 0\right)(\forall \psi)$.

Maximizando em relação a $\psi$ e introduzindo-se $X^{\prime}=X-A / \gamma^{\prime} \operatorname{sen} \varphi \quad$ e $K^{\prime}=K-A / \gamma^{\prime} \operatorname{sen} \varphi$ na expressão resultante obtém-se, respectivamente:

$\left((K-X)^{2}+4 \operatorname{sen} \theta^{2}\right)^{1 / 2}+\frac{2 A}{\gamma^{\prime}} \leq(K+X) \operatorname{sen} \varphi, \quad\left(\forall x_{2} \leq 0\right)$.

$\left(\left(K^{\prime}-X^{\prime}\right)^{2}+4 \operatorname{sen} \theta^{2}\right)^{1 / 2} \leq\left(K^{\prime}+X^{\prime}\right) \operatorname{sen} \varphi, \quad\left(\forall x_{2} \leq 0\right)$.

Considera-se que a existência de uma função $K\left(x_{2}\right)$ que verifique (45) é equivalente aquela de uma função $K^{\prime}\left(x_{2}\right)$ que verifique (46) (Dormieux, 1989). Após desenvolvimento desta última expressão, obtém-se o trinômio em $K^{\prime}$ :

$K^{\prime 2} \cos ^{2} \varphi-2 K^{\prime} X^{\prime}\left(1+\operatorname{sen}^{2} \varphi\right)+X^{\prime 2} \cos ^{2} \varphi+4 \operatorname{sen}^{2} \varphi \leq 0, \quad\left(\forall x_{2} \leq 0\right)$.

Assim, a existência de um real $K^{\prime}$ que verifique a inequação acima, a um $X^{\prime}$ dado, é assegurada se e somente se o discriminante deste trinômio satisfaz $\Delta \geq 0$ :

$X^{\prime}\left(x_{2}\right) \geq \operatorname{sen} \theta / \tan \varphi, \quad\left(\forall x_{2} \leq 0\right)$.

$X^{\prime}\left(x_{2}\right) \leq-\operatorname{sen} \theta / \tan \varphi, \quad\left(\forall x_{2} \leq 0\right)$.

Observa-se que se $X^{\prime}$ é negativo, (46) não pode ser verificada. Em contrapartida, se $X^{\prime}$ obedece (48) a existência de um escalar $K^{\prime}$ solução de (47) é assegurada e um campo de tensões $\underline{\underline{\sigma^{\prime}}}$ do tipo (43) compatível com a resistência pode ser construído. A partir de (48) uma condição suficiente de estabilidade do maciço pode ser encontrada:

$\frac{1}{x_{2}} \int_{0}^{x_{2}} i^{i r r}(\alpha) d \alpha \leq \frac{\gamma^{\prime}}{\gamma_{w}}\left(\frac{\operatorname{sen}(\varphi-\theta)-k p_{0} e^{k x_{2}} / \gamma^{\prime}}{\operatorname{sen} \varphi}\right), \quad\left(\forall x_{2} \leq 0\right)$.

onde foram utilizadas as seguintes relações introduzidas anteriormente: $X^{\prime}=X-A / \gamma^{\prime} \operatorname{sen} \varphi$, $X=\cos \theta+Q\left(x_{2}\right)$ e $A\left(x_{2}\right)=k p_{o} \mathrm{e}^{k x_{2}}$. Considerando as condições impostas sobre a função $\underline{i}^{i r r}$ 
mostra-se que o valor máximo do termo à esquerda da desigualdade acontece em $x_{2}=0$ e a condição suficiente de estabilidade, para $\gamma_{w} i^{i r r}$, fica definida da seguinte forma:

$$
\gamma_{w} i^{i r r}(0) \leq \gamma^{\prime}\left(\frac{\operatorname{sen}(\varphi-\theta)-k p_{0} / \gamma^{\prime}}{\operatorname{sen} \varphi}\right) .
$$

\subsection{Abordagem cinemática}

Considera-se a família de campos de velocidade virtual $\underline{V}^{\alpha \beta l}$ dependente dos três parâmetros geométricos $\alpha, \beta$ e $l$, como mostrado na figura 5 .
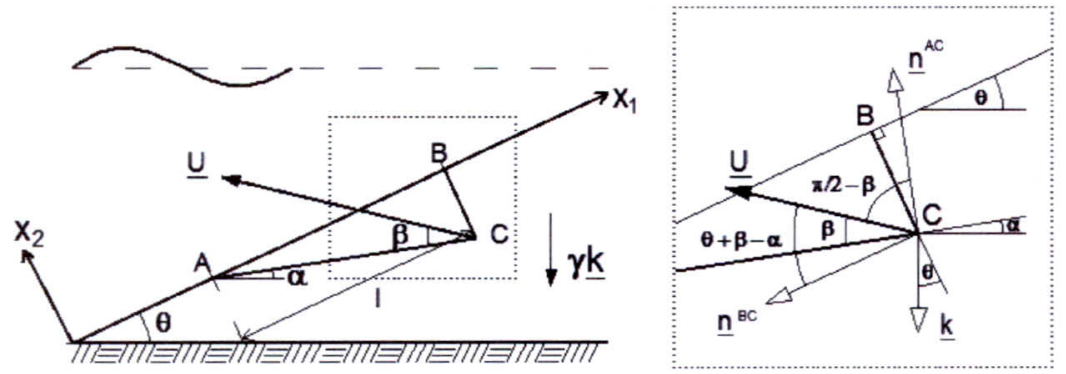

Figura 5 - Campo de velocidade virtual dependente de três parâmetros $\alpha, \beta$ e $l$.

O bloco triangular $\mathrm{ABC}$ está submetido a uma translação definida pela velocidade uniforme $\underline{U}$. No resto do maciço, a velocidade virtual é igual à zero. Os segmentos $\mathrm{AC}$ e $\mathrm{BC}$ constituem as linhas de descontinuidade do campo de velocidade. De início, os parâmetros $\beta$ e $l$ não estão submetidos a restrições, enquanto que o ângulo $\alpha$ está sujeito a seguinte condição geométrica:

$\alpha \in\} \pi / 2+\theta, \theta]$.

A potência resistente máxima consiste na soma das contribuições relacionadas a cada linha de descontinuidade do campo de velocidade, isto é, $A C$ e $B C$ :

$P_{r m}\left(\underline{V}^{\alpha \beta l}\right)=\int_{A C} \pi(\underline{n}, \underline{U}) d l+\int_{B C} \pi(\underline{n}, \underline{U}) d l$.

onde $\underline{n}$ em cada uma das integrais é o vetor unitário normal ao segmento AC e ao segmento $\mathrm{BC}$, respectivamente. Para o material de Coulomb sem coesão, o cálculo da função $\pi(\underline{n}, \underline{U})$ fornece:

$\pi(\underline{n}, \underline{U})=+\infty \quad$ se $\underline{U} \cdot \underline{n} \leq \operatorname{sen} \varphi$

$\pi(\underline{n}, \underline{U})=0 \quad$ se $\underline{U} \cdot \underline{n} \geq \operatorname{sen} \varphi$

onde "." denota produto escalar e $U$ é um escalar positivo.

A abordagem cinemática fornecerá resultados não-triviais somente se a função $\pi(\underline{n}, \underline{U})$ permanecer finita sobre os segmentos AC e BC. Para que isto ocorra, $\alpha$ e $\beta$ devem obedecer às seguintes condições:

CILAMCE 2015

Proceedings of the XXXVI Iberian Latin-American Congress on Computational Methods in Engineering Ney Augusto Dumont (Editor), ABMEC, Rio de Janeiro, RJ, Brazil, November 22-25, 2015 


$$
\begin{gathered}
\varphi \leq \beta \leq \pi-\varphi \\
-\pi / 2+\varphi \leq \beta+\theta-\alpha \leq \pi / 2-\varphi
\end{gathered} .
$$

onde $\theta=0$ no caso de fundo do mar plano horizontal. Observando que $\alpha \leq \theta$, verifica-se que essas duas desigualdades duplas se resumem a seguinte:

$\varphi \leq \beta \leq \pi / 2-\varphi+\alpha-\theta$.

Nota-se por $\mathscr{D}$ o domínio do plano $(\alpha, \beta)$ definido pelas condições (52) e (56). Observa-se que este domínio nunca é vazio pois ele contém a dupla $(\theta, \varphi)$. O teorema cinemático fornece então uma família de condições necessárias de estabilidade sob a forma seguinte:

$$
(\forall(\alpha, \beta) \in \mathscr{D}) \quad(\forall l \geq 0), \quad P_{e x t}\left(\underline{V}^{\alpha \beta l}\right) \leq 0 .
$$

A quantidade $P_{e x t}\left(\underline{V}^{\alpha \beta l}\right)$ representa a soma das contribuições das componentes da densidade volumétrica das forças externas, onde $\gamma_{w} \underline{i}=\gamma_{w} \underline{i}^{e}+\gamma_{w} \underline{i}^{i r r}$ :

$$
P_{e x t}\left(\underline{V}^{\alpha \beta l}\right)=\int_{A B C} \gamma^{\prime} \underline{k} \cdot \underline{U} d S+\int_{A B C} \gamma_{w} \underline{i}^{e} \cdot \underline{U} d S+\int_{A B C} \gamma_{w} \underline{i}^{i r r} \cdot \underline{U} d S .
$$

onde "." denota produto escalar e $d S$ é o diferencial de área, sendo $\mathrm{S}$ a superfície do triângulo ABC.

Uma vez que as grandezas vetoriais envolvidas no cálculo da potência externa são as mesmas nas análises do fundo plano horizontal e inclinado, o cálculo a seguir será desenvolvido para a situação mais abrangente, com $\theta \neq 0$. Utilizando-se $\theta=0$ obtêm-se respostas relativas ao fundo plano horizontal. As grandezas vetoriais são dadas por:

$$
\begin{aligned}
& \gamma^{\prime} \underline{k}=-\gamma^{\prime}\left(\operatorname{sen} \theta \underline{e_{1}}+\cos \theta \underline{e_{2}}\right) \\
& \gamma_{w} \underline{i}^{e}=-A\left(x_{2}\right)\left(\cos \psi \underline{e_{1}}+\operatorname{sen} \psi \underline{e_{2}}\right), \quad \psi=k x_{1}+\omega t, \quad A\left(x_{2}\right)=p_{0} k \mathrm{e}^{k x_{2}} . \\
& \gamma_{w} \underline{i}^{i r}=i^{i r r}\left(x_{2}\right) \underline{e_{2}} \\
& \underline{U}=U\left(-\cos (\theta+\beta-\alpha) \underline{e_{1}}+\operatorname{sen}(\theta+\beta-\alpha) \underline{e_{2}}\right)
\end{aligned}
$$

A potência externa fica definida da seguinte forma:

$$
P_{e x t}=U S\left[\gamma^{\prime} \operatorname{sen}(\alpha-\beta)+\frac{1}{S} \int_{A B C} A\left(x_{2}\right) \cos (\psi+\theta+\beta-\alpha) d S+\gamma_{w} \operatorname{sen}(\theta+\beta-\alpha) i_{\alpha d}^{i r r}\right] .
$$
onde $i_{o l}^{i r r}=\frac{1}{S} \int_{A B C} i^{i r r} d S$.

Substituindo a expressão acima na condição de estabilidade $P_{e x t}\left(\underline{V}^{\alpha \beta l}\right) \leq 0$ encontra-se a condição necessária de estabilidade:

$$
\begin{aligned}
& \gamma_{w} \operatorname{sen}(\theta+\beta-\alpha) i_{\alpha l}^{i r r} \leq \gamma^{\prime} \operatorname{sen}(\beta-\alpha)-\frac{1}{S} \int_{A B C} A\left(x_{2}\right) \cos (\psi+\theta+\beta-\alpha) d S . \\
& (\forall \psi \in[0,2 \pi]) \quad(\alpha, \beta) \in \mathcal{D} \quad(\forall l \geq 0)
\end{aligned}
$$


Considerando dados $\gamma^{\prime}$ e $A\left(x_{2}\right)$, deseja-se extrair a condição mais restritiva a ser verificada pela função $i_{o d}^{i r r}\left(x_{2}\right)$ a partir desta família de desigualdades. Com (II) e (56) observa-se que os escalares $\operatorname{sen}(\theta+\beta-\alpha)$ e $i_{\alpha d}^{\text {irr }}$ são positivos. A escolha do comprimento 1 deve ser otimizada de forma que o lado direito da desigualdade (61) seja minimizado e o lado esquerdo maximizado. Observando que para $\forall \psi \in[0,2 \pi]$ (Dormieux et al., 1993):

$$
\frac{1}{S} \int_{A B C} A\left(x_{2}\right) \cos (\psi+\theta+\beta-\alpha) d S \geq \min _{\psi}\left(\lim _{l \rightarrow 0} \frac{1}{S} \int_{A B C} A\left(x_{2}\right) \cos (\psi+\theta+\beta-\alpha) d S\right)=k p_{0}
$$
1989):

E que a função $i^{\text {irr }}$ é, para um dado valor de $\alpha$, decrescente em função de 1 (Dormieux,

$(\forall l \geq 0), \quad i_{\alpha l}^{i r r} \leq \lim _{l \rightarrow 0} i_{\alpha l}^{i r r}=i^{i r r}(0)$

Tomando o limite de (61) quando $l \rightarrow 0$ e otimizando a escolha de $\alpha$ e $\beta$, obtém-se a condição necessária de estabilidade:

$\gamma_{w} i^{i r r}(0) \leq \gamma^{\prime}\left(\frac{\operatorname{sen}(\varphi-\theta)-k p_{0} / \gamma^{\prime}}{\operatorname{sen} \varphi}\right)$

Nota-se que esta expressão é idêntica à condição suficiente de estabilidade (51) obtida pela abordagem estática. Assim esta desigualdade representa a condição necessária de estabilidade mais restritiva que pode ser fornecida pela abordagem cinemática.

\subsection{Delimitação da carga limite}

É interessante rearranjar (64) a fim de observar o papel das duas contribuições de densidade volumétrica da força de fluxo:

$$
\left[\frac{\gamma_{w}}{\gamma^{\prime}} \frac{\operatorname{sen} \varphi}{\operatorname{sen}(\varphi-\theta)}\right] i^{i r r}(0)+\left[\frac{1}{\operatorname{sen}(\varphi-\theta)}\right] \frac{k p_{0}}{\gamma^{\prime}} \leq 1
$$

A inequação (65) faz aparecer claramente 2 parâmetros que quantificam a intensidade do carregamento ao qual o maciço é submetido. O parâmetro $Q_{1}=k p_{0} / \gamma^{\prime}$ constitui uma medida direta da intensidade do carregamento. O parâmetro $Q_{2}=i^{\text {irr }}(0)$ leva em conta as deformações volumétricas irreversíveis causadas pelo carregamento. $\mathrm{O}$ domínio $\mathrm{K}$ dos valores potencialmente suportáveis destes dois parâmetros é o triângulo representado na Fig. 6.

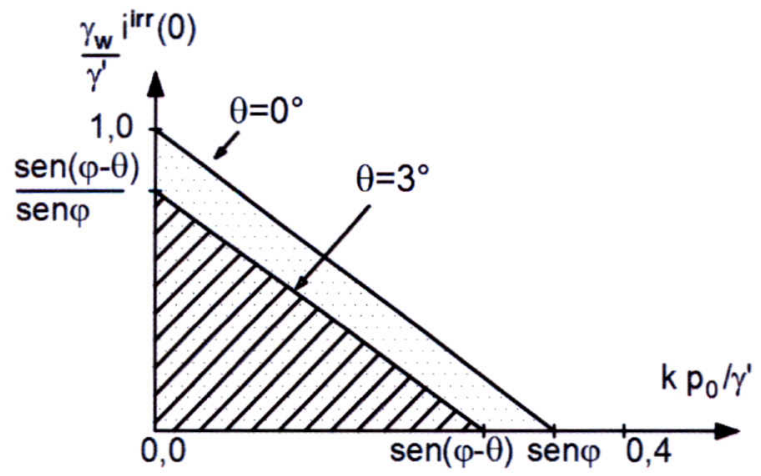

Figura 6 - Domínio K dos valores potencialmente suportáveis dos parâmetros (Q1, Q2). 
Para interpretar fisicamente o resultado obtido, introduz-se a quantidade:

$$
i^{c r}=\frac{\gamma^{\prime}}{\gamma_{w}} \frac{\operatorname{sen}(\varphi-\theta)}{\operatorname{sen}(\varphi)}
$$

A qual representa o valor extremo do gradiente hidráulico perpendicular ao declive e orientado para sua superfície superior. Ela constitui uma generalização do conceito de gradiente hidráulico crítico $\gamma^{\prime} / \gamma_{w}$ comumente encontrado na mecânica dos solos para estudo da estabilidade devido a um fluxo de água ascendente, levando em conta o efeito desfavorável da inclinação do maciço. Contudo, sob carregamento da onda, forças de fluxo de direção variável se superpõe às forças de fluxo perpendiculares ao declive. Assim, a instabilidade pode aparecer ciclicamente desde que o ponto representativo do carregamento sobre o plano $\left(\mathrm{Q}_{1}, \mathrm{Q}_{2}\right)$ atinja a fronteira de $\mathrm{K}$, mesmo se $i^{i r r}(0)$ é situado abaixo do gradiente crítico $i^{\text {cr }}$.

Nas análises de estabilidade propostas por Ishihara \& Yamazaki (1984) e Rahman \& Jaber (1986), as quais preocuparam-se com o risco de liquefação, a aparição de instabilidade no maciço é caracterizada por um critério equivalente a: $i^{i r r}(0)=\gamma^{\prime} / \gamma_{w}$. Essa definição de instabilidade além de não levar em conta a declividade do maciço parece ser menos restritiva que (69), pois o papel desfavorável de $\mathrm{Q}_{1}$ não é levado em conta.

Do ponto de vista prático, a predição da amplitude da onda $\mathrm{p}_{0}$ que induz a instabilidade do maciço submarino necessita a avaliação prévia do gradiente hidráulico na superfície, a partir das propriedades físicas e geométricas do maciço.

\section{CONCLUSÕES}

A questão da estabilidade de maciços submarinos submetidos ao carregamento da onda, modelado conforme a teoria linear, foi examinada para um solo coesivo e um solo granular no âmbito da teoria da Análise Limite, baseada em condições de deformações planas. Na seção 3 foram identificadas as condições de estabilidade para o maciço coesivo e, para modelagem da resistência deste solo fino, o critério de ruptura de Tresca linear foi empregado. Obervou-se que, do ponto de vista da estabilidade e considerando a solicitação modelada pela teoria linear, a declividade $\mathrm{H} / \mathrm{L}$ aparece como o parâmetro característico da onda e o domínio de valores destes parâmetros que provocam a instabilidade foi determinado de forma exata. Com relação ao efeito da inclinação, verificou-se que a estabilidade de um maciço inclinado segundo um ângulo $\theta$ com a horizontal pode ser deduzida daquela de um maciço horizontal constituído pelo mesmo material através da redução do gradiente de coesão no valor de $\gamma^{\prime} \operatorname{sen} \theta$. A seção 4 apresentou um método de análise da estabilidade de um maciço granular. Em razão do acoplamento entre o fluido intersticial e o esqueleto, uma modelagem do comportamento, além do critério de ruptura, foi necessária para caracterizar o carregamento em tensões efetivas. Tratou-se de determinar as forças de fluxo atuantes sobre o esqueleto sólido de origem reversível e irreversível e, com um cálculo aproximado destas forças, foi possível colocar em evidência seus papéis críticos. Em termos da função i ${ }^{\text {irr }}$ foi possível obter a condição necessária de estabilidade mais restritiva.

De forma geral, uma diferença fundamental aparece no que se refere à definição do carregamento. No caso do solo coesivo, este é determinado completamente pelas características físicas da onda de projeto e a escolha do modelo da onda. Enquanto que, para um solo granular, a introdução de informações suplementares sobre a contractância do material é indispensável para transmitir os parâmetros da onda à definição das forças externas 
atuantes sobre o maciço. Essa diferença tem origem na escolha dos critérios de ruptura distintos, em função da natureza do solo. Em razão da fase preliminar destinada a definição do carregamento, o estudo do caso granular pode parecer mais complexo. Mas a simplicidade relativa do caso coesivo é enganosa, uma vez que ela mascara a complexidade da determinação do parâmetro "coesão não-drenada", que concentra toda informação disponível sobre o material.

\section{REFERENCES}

Coussy, O., 2004. Poromechanics. John Wiley \& Sons Ltd.

De Buhan, P.; Cuvillier, P.; Dormieux, L.; et al., 1999. Face stability of shallow circular tunnels driven under the water table: A numerical analysis. International Journal for Numerical and Analytical Methods in Geomechanics, vol. 23, n. 1, pp. 79-95.

Dormieux, L., 1988. Stability of a purely cohesive sea bed soil under wave loading. Géotechnique, vol. 38, n. 1, pp. 121-123.

Dormieux, L., 1989. Influence de la houle sur la stabilité d'un massif sous-marin. Thèse Dr, École Nationale des Ponts et Chaussées, Paris.

Dormieux, L. 1992. Stability Analysis of a seabed subjected to wave loading. Mechanics Research Communications, vol. 19, n. 4, pp. 325-331.

Dormieux, L.; Auriault , J.-L.; Coussy, O., 1993. Pore pressures generation in a seabed subjected to wave loading. Eur. J. of Mech., A/Solids, vol. 12, n. 6, pp. 773-801.

Dormieux, L.; Coussy, O., 1991. Stability of a sedimentary half-space subjected to an arbitrary pressure distribution. Eur. J. Mech., A/Solids, vol. 10, n. 4, pp. 371-394.

Dormieux, L.; Delage, P., 1988. Effective stress response of a plane sea-bed under wave loading. Géotechnique, vol. 38, n. 3, pp. 445-450.

Ishihara, K.; Yamazaki, A., 1984. Analysis of wave-induced liquefaction in seabed deposits of sand. Soils and Foundations, vol. 24, n. 3, pp. 85-100.

Rahman; Jaber, C. L., 1986. A simplified drained analysis for wave-induced liquefaction in ocean floor sands, Soils and Foudations, vol. 26, n. 3, pp. 57-68.

Saada, Z.; Maghous, S.; Garnier, D., 2012. Stability analysis of rock slopes subjected to seepage forces using the modified Hoek-Brown criterion. International Journal of Rock Mechanics and Mining Sciences, vol. 55, pp. 45-54.

Salençon, J., 1983. Calcul a la rupture et analyse limite. Presses de l’E.N.P.C., Paris.

Salencon, J., 1990. An introduction to the yield design theory and its application to soil mechanics. European Journal of Mechanics A/Solids, vol. 9, n. 5, pp. 477-500.

Salencon, J., 2013. Yield Design. John Wiley \& Sons, Inc.

Seed, H. B.; Martin, P. P.; Lysmer, J, 1976. Pore water pressure changes during soil liquefaction. Journal of the Geotech. Eng. Div., ASCE, GT4, 102, pp. 323-346. 